\title{
ON A NEW EQUIVALENCE OF COEFFICIENT CONDITIONS AND APPLICATIONS
}

\section{LÁSZLÓ LEINDLER}

Abstract. We show that if the sequence $\left\{\kappa_{n}\right\}$ is quasi geometrically increasing, then a so-called block-condition

$$
\sum_{m=0}^{\infty} \kappa_{m}\left(\sum_{n=v_{m}+1}^{v_{m+1}}\left|c_{n}\right|^{q}\right)^{p / q}<\infty, \quad 0<p<q,
$$

for every $\left\{v_{m}\right\}$ is equivalent to the following two conditions

$$
\sum_{n=1}^{\infty}\left|c_{n}\right|{ }^{q} \mu_{n}<\infty \text { and } \sum_{n=1}^{\infty} \kappa_{n}\left(\frac{\kappa_{n}}{\mu_{v_{n}+1}}\right)^{\frac{p}{q-p}}<\infty,
$$

where $\left\{\mu_{n}\right\}$ is a nondecreasing sequence. sented.

Applications to absolute $|C, \alpha|$-summability of general orthogonal series are also pre-

Mathematics subject classification (1991): 26D15, 40F05, 40G05, 42C15.

Key words and phrases: Inequalities for sums, absolute summability, orthogonal series.

\section{REFERENCES}

[1] G. H. Hardy, J. E. LitTlewood And G. Pólya, Inequalities, Cambridge University Press, Cambridge (1959).

[2] L. LEINDLER, Über die absolute Summierbarkeit der Orthogonalreihen, Acta Sci. Math., Szeged 22 (1961), 243-268.

[3] _ Über unbedingte Konvergenz der Orthogonalreihen mit strukturellen Bedingungen, Studia Math. 23 (1963), 113-117.

[4] _ Über einen Äquivalenzsatz, Publ. Math., Debrecen 12 (1965), 213-218.

[5] On relations of coefficient-conditions, Acta Math. Acad. Sci. Hungar. 39 (1982), 409-420.

[6] On equivalence of coefficient conditions with applications, Acta Sci. Math., Szeged 60 (1995), 495-514.

[7] - On equivalence of coefficient conditions and application, Math. Ineq. \& Appl., Zagreb 1 (1998), 41-51.

[8] Y. OKUYAMA AND T. TSUChIKURA, On the absolute Riesz summability of orthogonal series, Analysis Math. 7 (1981), 199-208.

[9] W. OrLICZ, Zur Theorie der Orthogonalreihen, Bull. Acad. Polonaise (1927), 81-115.

[10] V. TOTIK AND I. VINcZE, On relations of coefficient conditions, Acta Sci. Math., Szeged 50 (1986), 93-98. 